\title{
RELACIÓN DE LA COMUNICACIÓN, LA EDUCACIÓN Y LAS NUEVAS TECNOLOGÍAS EN LA UNIVERSIDAD PEDAGÓGICA NACIONAL**
}

\begin{abstract}
Resumen
Este texto se realizó con apartes extractados de la investigación sobre Estado del Arte de la comunicación y las nuevas tecnologías en la Universidad Pedagógica Nacional, realizado en 2001 por el autor, para el Colegio Académico de Comunicación y Educación - CACE- de la UPN.

Contiene nociones y conceptos hallados mediante revisión bibliográfica y consultas efectuadas a profesores $e$ investigadores, de proyectos y programas de la Universidad, sobre la relación de la comunicación, la práctica pedagógica y el uso de tecnologías de la información en la Universidad Pedagógica.

El propósito del escrito aquí presentado es dar a conocer, de forma descriptiva, aspectos y características de comunicación y uso de tecnologías en espacios académicos e investigativos de la Universidad y formular el interrogante de: ¿Cuál es, o se cree que es, el papel de la comunicación y las nuevas tecnologías de la información en los procesos educativos de la UNP? Pregunta que se resolvió a través de citas de textos compilados en el proceso de consulta con miembros de la comunidad universitaria y específicamente en la prospectiva y conclusiones.
\end{abstract}

Palabras clave. Universidad Pedagógica Nacional, educación, comunicación, información, nuevas tecnologías, investigación.

\section{Abstract}

The text was carried out with extracted parts of the research about Communication Art State and the new technologies in the National Pedagogic University (UPN), that was make by the author in 2001, for the Communication and Education Academic School - CACE - of the UPN.

It contains notions and concepts found by means of bibliographical reviews and questions made to professors and researchers, of both projects and programs of the University, about the communication, pedagogic practice and information technologies use relationship.

\footnotetext{
*Especialista en Educación-Comunicación. Profesor Universidad Pedagógica Nacional. E-mail: groncancio@uni.pedagogica.edu.co "Este artículo hace parte de la investigación sobre Estado del arte de la comunicación y las nuevas tecnologías en la Universidad Pedagógica Nacional realizado en el año 2001. Recibido el 5 de noviembre de 2003 y aprobado el 14 de noviembre de 2003.
} 
The purpose of this paper is show, in a descriptive way, some aspects and characteristics of communication and technologies use in academic and research spaces of the University and to made the question ¿ Which is, or what is believed that is it, the roll of the communication and new information technologies in the educative processes of the UPN? This question was solved through appointments of text compiled in the consulting process with members of the university community and specifically in the prospective and conclusions.

Key words. National Pedagogic University, education, communication, information, new technologies, research.

\section{PRESENTACIÓN}

El presente trabajo consiste en la compilación y en la presentación, principalmente descriptiva, de algunos estudios sobre aspectos y características que de la comunicación y uso de tecnologías tienen programas y proyectos de la UPN. Es decir, es una síntesis descriptiva de la revisión bibliográfica y apuntes obtenidos en la selección de textos efectuada en otro estudio realizado sobre el mismo tema': Relación de la comunicación, la educación y las nuevas tecnologías en la Universidad Pedagógica Nacional.

Este material tiene como objetivo dar a conocer nociones y conceptos que sobre el tema expresan profesores e investigadores de la Universidad, en la perspectiva de establecer posibles relaciones de interdisciplinariedad y así coadyuvar a la construcción de comunidades académicas en interacción con el entorno educativo y con el contexto socioeconómico y político de la nación colombiana.

El problema a indagar, implícito en el texto, puede anunciarse como: ¿Cuál es, o se cree que es, el papel de la comunicación y las nuevas tecnologías de la información en los procesos educativos de la UNP? Pregunta que se irá respondiendo a medida que se citan los textos com-

\footnotetext{
${ }^{1}$ Compendio inédito del estado del arte de la investigación en comunicación y tecnologías de la información en la UPN. realizado para el Colegio Académico de Comunicación y Educación -CACE- de la Universidad Pedagógica Nacional, por el autor en 2002.
}

pilados y específicamente en las observaciones y conclusiones. Subyace también en la presentación de este articulo, la intención de fortalecer la promoción e interacción del conocimiento y praxis de la comunicación en sus diversas formas y expresiones, de tal manera que se promueva la participación democrática de la comunidad pedagógica en el devenir cultural de la universidad y en la búsqueda de soluciones a los conflictos políticos y sociales del país.

El texto plantea diferentes apreciaciones sobre el uso de las nuevas tecnologías de la información; describe criterios de cómo se concibe la relación de la comunicación con la práctica pedagógica; finalmente enuncia algunas conclusiones y prospectiva. Este articulo es un reducido extracto de apartes y citas de la investigación sobre Estado del Arte de relaciones entre la comunicación y las nuevas tecnologías en la Universidad Pedagógica Nacional, realizado por el autor, en el marco de las labores del entonces existente Colegio Académico de Comunicación y Educación -CACE- en el año 2001.

\section{INTRODUCCIÓN}

Dado que los procesos de globalización e irrupción abrupta de las nuevas tecnologías de la información, en lo más cotidiano de nuestras vidas, hacen pensar que nos dirigimos hacia un mundo homogéneo en donde reinará el "pensamiento único", se requiere que asumamos sus sentidos de manera diferente y pen- semos en la posibilidad de actuar localmente sin negarnos como habitantes del mundo global.

En tal sentido cabe preguntarnos: ¿Es inevitable considerar que asistimos al reinado de la homogeneidad en un mundo donde confluye la diversidad cultural, política, ideológica? En este marco, ¿Cuál sería el papel de la escuela y por ende, del maestro, en este nuevo contexto de relaciones ciencia-tecnologíasujeto?

Ante estas cuestiones se requiere partir de reconocer el hecho del aprendizaje como un proceso de construcción colectiva de acuerdos sobre explicaciones posibles, procedimientos y análisis de resultados, además de las competencias de carácter lógico o técnico, es preciso asumir actitudes en relación con la interacción social para ampliar las posibilidades de expresión, argumentación, comunicación y construcción de consensos.

Entonces, una investigación que tiene que ver con la comunicación y la tecnología de la información parte del postulado de la no "neutralidad" del conocimiento científico, lo cual implica, entre otros aspectos, que "cualquier ciencia, como producto cultural humano, tiene igualmente un propósito humano concreto $\mathrm{y}$, por ende, carga implícitamente con los prejuicios y valores presentes en la clase científica como grupo" (Fals Borda y Rahman, 1991)2, en con-

${ }^{2}$ Citado en Proyecto de Investigación: "Conflicto, Pedagogía y Cultura Democrática", realizado por el equipo de la Maestría de 
traposición a la posición de un conocimiento científico "objetivo", exacto e imparcial propio de la ciencia positivista.

En esta perspectiva es pertinente que en un estudio de la comunicación y la tecnología se analicen las relaciones de los actores educativos, tomando en cuenta la mutua interdependencia de prácticas y representaciones inmersas en la estructura social de la Universidad.

\section{COMUNICACIÓN-EDUCACIÓN, MEDIOS Y TECNOLOGÍAS DE LA INFORMACIÓN}

El capítulo contiene una serie de citas de textos y comentarios expresados por varios autores encontrados en la búsqueda bibliográfica, con miras a proporcionar un panorama de concepciones en torno a la relación de la educación, la comunicación y los medios; selección de apartes que por sí mismos muestran la diversidad de concepciones y posiciones que sobre el tema existen en la Universidad Pedagógica y que son de nuestro interés enunciar.

Así encontramos, por ejemplo: Que la categoría histórica-cultural, mencionada en la obra de Huergo (1999), permite encontrar la existencia de una sustitución o transferencia de espacios comunicativos y educativos, lo que puede llevar a replantear la relación de educación-comunicación y uso de las tecnologías de la información: "Este desplazamiento de la hegemonía en la formación de las conciencias, tiene relación con el paso de las culturas orales, a la lógica escritural y a la hegemonía audiovisual. Necesitamos comprender estos tres momentos históricos y socioculturales como coexistentes en la actualidad latinoamericana, y coexistiendo de manera no armónica sino conflictiva. Hay un cúmulo

Educación Comunitaria. Departamento de Postgrados, Facultad de Educación. UPN. Bogotá, 2002. de estrategias que tienden a una armonía en la comunicación para favorecer la tarea educativa, que en general se ha sustentado en el desplazamiento hacia el "receptor" y el desplazamiento de la concepción "bancaria" hacia el feed-bach o retroalimentación"3.

Introducir el tema de la comunicación y la cultura, en lo que tiene que ver con la educación, para Bustamante y Vásquez (2002) significa, no sólo ampliar el horizonte de los estudios educativos, sino también ensanchar los terrenos de la pedagogía, cargándola de nuevas preguntas e inquietudes en relación con la cultura contemporánea. "En la educación, la comunicación abre un nuevo campo de problemas que permiten ubicar distancias y cercanías entre los discursos pedagógicos y las prácticas comunicativas, sus tensiones y contradicciones. En este campo, confrontar las prácticas comunicativas de los actores escolares con los hechos educativos y culturales, constituye crear un nuevo escenario para el abordaje pedagógico"4.

En esta perspectiva, continuan Bustamante y Vásquez; "la relación comunicación-educación saca a flote un inédito campo de problemas, cuyas manifestaciones, a veces difusas e intangibles, escapan a las miradas ortodoxas que sólo ven evidencias en los hechos educativos y comunicativos a partir de los cuales puedan ejercer control sobre el sa-

\footnotetext{
${ }^{3}$ Huergo, Jorge Alberto y Osin, Luis. Comunicación, Humanismo y nuevas Tecnologías en el espacio escolar. CACE-UPN, 1999. 4 En el "Observatorio Pedagógico de Medios" los profesores Guillermo Bustamante y Tomás Vásquez asumen la relación comunicación-educación como un campo de problemas comunes a la educación y a la comunicación, pero a su vez consideran que constituye un área particular de problemas que se generan en ese cruce interdisciplinar. El campo comunicación-educación es abordado desde las mediaciones culturales lo que posibilita introducirle un sentido histórico a la problemática y "sacarla" del abstraccionismo y del instrumentalismo con los que por lo general se asume.
}

ber y la organización escolar... De este modo, cruzar la comunicación con la educación es abrir un foco de análisis a los fenómenos culturales y políticos que se reproducen y atraviesan el espacio social y educativo, es decir, observar la cultura escolar en toda su complejidad y sus relaciones con otras culturas con las que se encuentra, produciéndose lo que hoy se ha denominado encrucijada de cultura"5.

Por otra parte, encontramos que Ávila (1999) enuncia una estrategia o función, como conectador o entrelace de relación educación, comunicación y tecnologías de la información: "La interactividad permite el desarrollo de procesos de comunicación e intercambio entre los sujetos rompiendo barreras temporales y espaciales, por tanto, el medio está jugando un papel socializador"6.

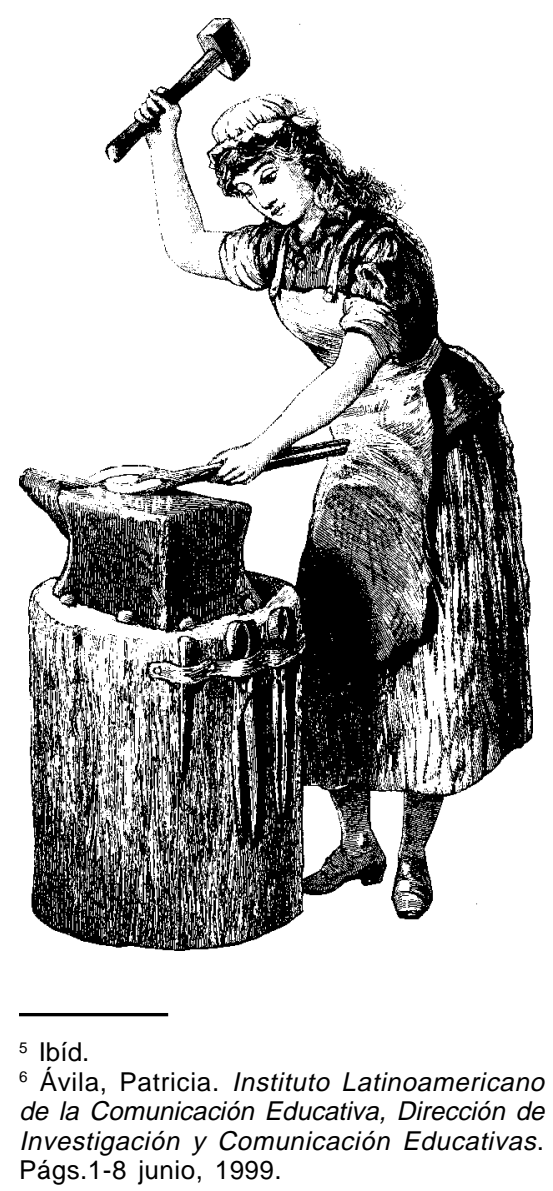


En experiencias sobre investigación y docencia en el desarrollo de proyectos de la UPN, se plantea como indicativo de los aportes de la relación, educación y uso crítico de nuevas tecnologías que: "La integración de flujos curriculares destinados a fortalecer el desarrollo de competencias comunicativas, la integración de procesos de formación para el desarrollo de habilidades en el dominio de los medios de comunicación y las nuevas tecnologías de la información deben ser concebidos en un nuevo modelo de enseñanzaaprendizaje que propicie de manera real el desarrollo de la capacidad de adaptación del estudiante al cambio constante, al acceso a nuevas fuentes de información, al desarrollo de criterios para usar esta información y al desarrollo de la inteligencia, la reflexión y el pensamiento crítico. Si estos objetivos no se producen, la integración de la comunicación, los medios y las nuevas tecnologías en los procesos de renovación curricular y de acreditación no tiene mayor sentido"'.

Así mismo, Osin y Huergo, cuestionan la idea que prevalece con respecto a considerar que el uso de las tecnologías sería la solución al problema de la disparidad en el flujo informativo entre emisores y receptores de conocimiento, al expresar que: "La hegemonía tecnológicoinformacional produce una representación de la "comunicación para la educación" que contiene la creencia de que los avances tecnológicos se corresponden con una vida más feliz. La ilusión de que la "salida de la crisis" se producirá gracias a las altas tecnologías de la comunicación que, como observa Armand Mattelart (1984), cumplirían un pa-

\footnotetext{
${ }^{7}$ Relaciones y tensiones entre investigación e innovación en educación. Colección: Desarrollos en investigación en educación 1. (Compilación) Blanca Cecilia Barbosa Alonso. "El córtex digital: Posibilidades de desarrollos cognitivos en los nuevos espacios virtuales". Zambrano, Marco Fidel. Págs. 141174. Universidad Pedagógica Nacional. Simposio Nacional de Investigación Educativa, 1999.
}

pel similar al de la religión, en cuanto posibilidad de re-ligar o re-unir a la humanidad"8. De igual forma, el mejor aprendizaje, el desarrollo de habilidades superiores y actitudes positivas hacia el aprendizaje, afirman Zimra y Elad ${ }^{9}$, no están aseguradas por la disponibilidad y el uso de las tecnologías.

Las tecnologías no llegan más allá de los individuos, las organizaciones y las sociedades que las usan, continúa el texto citado y concluye... "La integración de medios y nuevas tecnologías en el espacio escolar no pueden entenderse como un problema exclusivo de infraestructura y dotación de equipos informáticos y de telecomunicaciones. Igualmente no pueden entenderse como creación improvisada de espacios informáticos destinados a la exposición simple y desestructurada a dispositivos informáticos. Esta visión reduccionista puede conducir a frustraciones profundas de la organización escolar con relación a los beneficios que los medios y la tecnología pueden generar en los procesos de enseñanza-aprendizaje"10.

Ahora bien, producto de la observación y clasificación de textos revisados, sobre tecnologías de la información, en la búsqueda realizada en algunos proyectos, programas e investigaciones de la UPN, se hallaron principalmente algunas tendencias y formas de ver la informática, posiciones e interpretaciones sobre el uso de las tecnologías de la información, (NTIC). Tales tendencias se pueden agrupar en tres concepciones o postulados:

Una primera posición que asume las NTIC como la panacea, lo máximo y más importante, casi lo único que podrá salvarnos o sacarnos del atra-

\footnotetext{
8 Op. cit. Osin, Luis; Huergo, Jorge Alberto. P. 176.

${ }^{9}$ Op. cit. Relaciones y tensiones entre investigación e innovación en educación. ${ }_{10}$ Ibid.
}

so. Esas visiones viven solo en función de las tecnologías, desconocen o subestiman las otras ciencias o saberes y exponen que el 'éxito' está determinado por la adquisición de la última moda en telemática $y$, aún cuanto esto mantenga la dependencia, económica y tecnológica del país, piensan sólo en copiar programas o, en el mejor de los casos, publican o experimentan en Software.

Es "una visión funcionalista económica, basada en un concepto de capital humano, para la alfabetización tecnológica, argumentando la necesidad de consumidores alfabetizados y de jóvenes tecnológicamente alfabetos equipados para entender y utilizar nuevas tecnologías en campos específicos"11.

Una segunda tendencia, todo lo critica y no produce nada, sataniza los medios y las NTIC, así como hace un siglo se rechazó la revolución industrial, trata de desconocer los actuales avances tecnológicos e ignora los usos benéficos que bien pue-

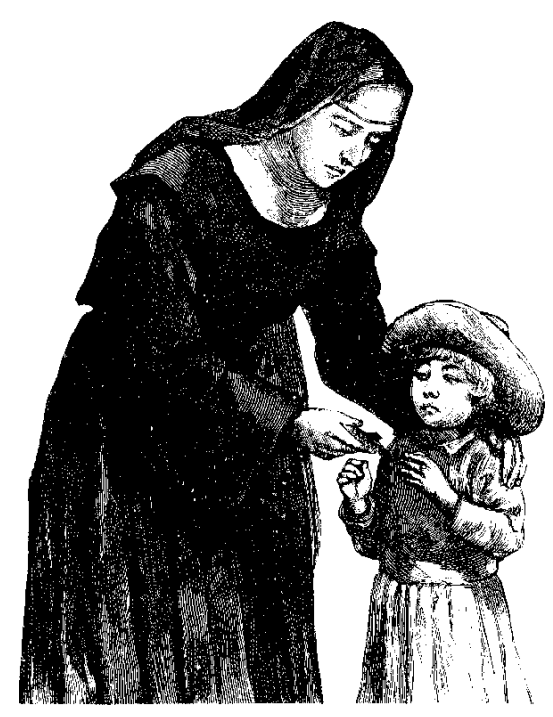

11 Citado en OECD Technology and the Economy. The Key Relationship.París. 1992, citado en GOEL,Ved.Scientific and Technological Literacy Meanings and Rationales - A Review Unesco. París, 1993. Págs. 37-38. 
den tener las tecnologías en los procesos educativos. En ese mismo sentido, se tiende a contribuir con la desmitificación de las reales posibilidades de la ciencia y la tecnología y las potencialidades de los seres humanos, por esto Botero (1993) afirma que "Se han fundado demasiadas ilusiones acerca de las posibilidades de la tecnología para resolver problemas contemporáneos acuciantes como, por ejemplo, el del deterioro ambiental. En general, el acervo del conocimiento científico y el desarrollo tecnológico en sí mismos no traerán las transformaciones que está reclamando la sociedad actual"12 ${ }^{2}$. La alfabetización en ciencia y tecnología debe entenderse, no como efecto de sus aplicaciones instrumentales sino, concluye Botero Lotero, como el contribuir a establecer las posibilidades de ellas frente al ser humano, en particular, y las comunidades, en general.

La tercera posición, que parte del contexto político, cultural y social, reflexiona sobre la incidencia de la globalización y el papel de las tecnologías en la implantación del modelo económico, para analizar los pro y contra de esos adelantos e innovaciones. Busca integrar, crítico-propositivamente, los desarrollos de la tecnología con base en los procesos productivos e intereses sectoriales, gremiales y de clase social específicos. Esta opción cuestiona los riesgos de tomar incondicionalmente la importación de las nuevas tecnologías de la información y la comunicación, NTIC; también, advierte la validez e importancia de usar esas tecnologías con base en nuestras propias necesidades y procesos educativos y culturales concretos.

\footnotetext{
${ }^{12}$ Botero Lotero, Amparo. "Alfabetización en ciencia y tecnología como parte integral de un proyecto de democratización y uso social de las telecomunicaciones". Memorias Seminario: "Uso social y democrático de las nuevas tecnologías". Universidad Pedagógica Nacional, UNESCO, Bogotá 1993, (pág. 41).
}

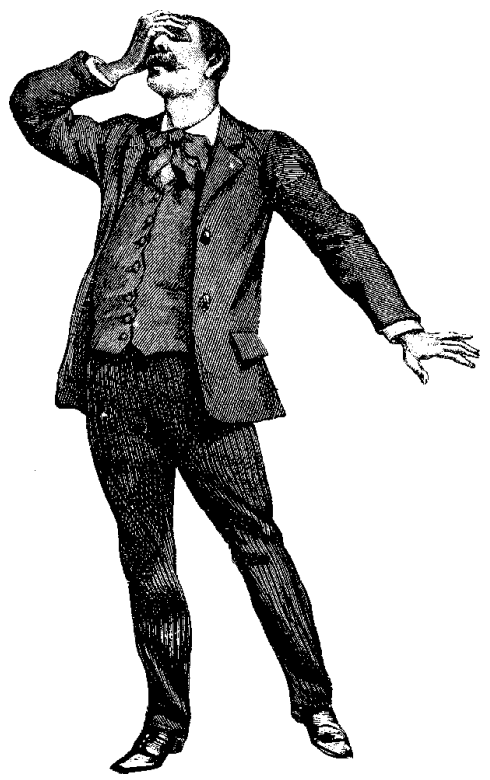

Resulta claro que no se trata de desconocer la importancia del uso de las tecnologías de la información en la educación, por el contrario, se considera que es necesario inscribirlas dentro de una concepción mucho más amplia de procesos comunicativos, económicos, sociales, políticos y culturales. Además de tener en cuenta el contexto social y el entorno educativo, hay que plantearse los enfoques y perspectivas que desde el sujeto social y las comunidades específicas se tienen y se requieren para un desarrollo autónomo, que trascienda la dependencia de los grandes consorcios que imponen sus tecnologías, para usufructo propio, profundizando el atraso histórico de nuestros pueblos. En este marco es imperativo colocar la ciencia y la tecnología al servicio de los sectores sociales que propenden por el rescate de la autodeterminación, soberanía e independencia en la perspectiva de que un mundo mejor es posible.

\section{EDUCACIÓN, PRÁCTICAS PEDAGÓGICAS Y USO DE TECNOLOGÍAS}

La escuela se configuró como institución destinada a reproducir las es- tructuras sociales establecidas, con una fuerte carga de disciplina, es decir, de organización racional de la vida social cotidiana, a la que se considera por lo menos no racional. "Esta tradición estratégica 'oficial' cobra en la actualidad una renovada fuerza, ahora ligada a los requerimientos de eficacia y eficiencia propios de los modelos tecnocráticos en educación"13.

Por ejemplo, en América Latina encontramos que: "Los relativos alcances que tuvo la estrategia del desarrollo y la modernización en estos países latinoamericanos, para los cuales el desempleo producido por la crisis demostró cuán falaz era la ecuación a más educación más prosperidad. Ni siquiera haberse preparado para la vida laboral garantiza ocupación, ni empleo, aun cuando así lo sigue diagnosticando quienes se han casado con esa concepción"14.

Las nuevas condiciones obligan a pensar en algo distinto, propone Nieto. "La Comunicación no es una unidad académica tradicional sino de enlace, que funciona de forma nodal, lo cual posibilita articular la relación Educación Comunicación, con otras fuentes de conocimiento y de trabajo educacional. De igual modo es clave comprender que los medios no son meras técnicas transmisoras. Los medios audiovisuales e informáticos son hoy como lo es el libro desde su aparición-actores culturales, así lo sean de una cultura distinta a la letrada"15. Se refiere Nieto, a la cultura en su sentido fuerte, antropológico, pues lo que se pro-

\footnotetext{
${ }^{13}$ Osin, Luis; Huergo, Jorge Alberto. "Comunicación, humanismo y nuevas tecnologías en el espacio escolar", CACE - UPN 1999, pp. 158-168.

${ }_{14}$ Martín-Barbero, Jesús, Herrán, María Teresa. En: Palimpsestos y recorridos de la comunicación en la educación, Bogotá, CACE UPN, 1999.

${ }^{15}$ Entrevista personal realizada por María Teresa Herrán al profesor Daniel Nieto de la Facultad de Bellas Artes de la UNP, para el libro Palimpsestos y recorridos de la comunicación en la educación. CACE-UPN, 1999.
} 


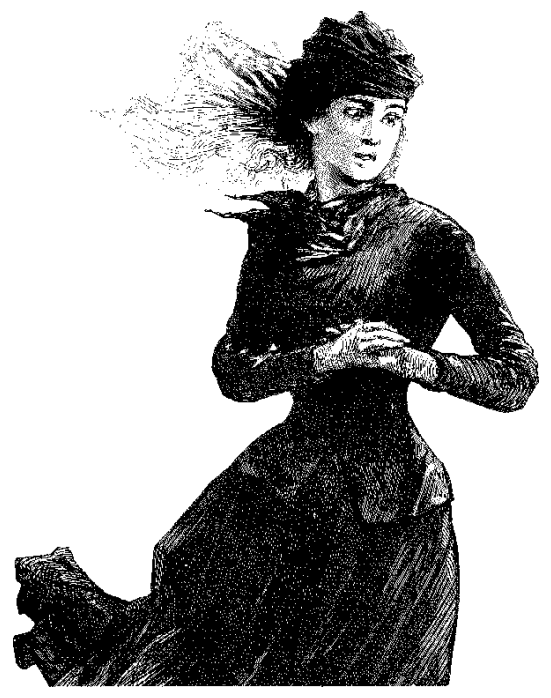

duce hoy en los medios son otros modos de percibir del espacio y el tiempo, nuevos modos de aprender y de saber.

De igual forma Huergo $(1999)^{16}$ ve allí, en la nueva relación de la educación con la comunicación, en cierta manera como alternativa a esas políticas impositivas, una estrategia de formación docente que busca el 'descongelamiento' de las prácticas pedagógicas a partir de la consideración de la docencia como una practica comunicacional, en un escenario de investigación acción, lo cual lleva a buscar, tanto en el ámbito comunicacional como en el educacional, una "autonomización del receptor" y abordar el controversial tema de las hegemonías académicas o de las luchas por el poder intelectual que no dejan de ser frecuentes entre las distintas disciplinas en Latinoamérica.

Cabe entonces la pregunta de investigación que formuló Ávila Muñoz: ¿De qué manera convergen la comunicación y la educación en el aprendizaje? El aprendizaje ya no es el mismo cuando está soportado con tecnologías duras; el diseño conceptual para introducir estas tec-

16 Op. cit. nologías al servicio de la educación es una tarea primordialmente pedagógico-comunicacional ${ }^{17}$.

En torno a la anterior pregunta de Ávila, es interesante dialogar con la reflexión que realizó Calvo: "Ante estos contextos los fines de la educación necesitan encaminarse hacia procesos formativos más que informativos. Las autopistas de la información estarán saturadas de datos al alcance de toda clase de usuarios y la producción de conocimiento sólo será posible a partir de rigurosos procesos mentales que puedan ponderar la información pertinente para los avances conceptuales en las diferentes disciplinas"18.

Desde una perspectiva de la modernidad, la práctica pedagógica es entendida en forma amplia como la innovación. "Esta debe permitir romper las rígidas estructuras tradicionales de la escuela: Verticalismo de las relaciones personales y pedagógicas, memorismo, autoritarismo, compartimentalización del conocimiento, aislamiento de la escuela de la vida, entre otros"19.

El panorama anterior remite a indagar cómo estamos realizando la práctica y si ¿estamos preparados los docentes, estudiantes y directivos universitarios para afrontar los cambios en el proceso educativo que estamos viviendo? Al respecto se plantea que "Hoy, al inicio del siglo XXI, está surgiendo un cambio trascendental en la educación con

\footnotetext{
17 "Aprendizaje con nuevas tecnologías: Paradigma emergente". Instituto Latinoamericano de la Comunicación Educativa. Dirección de Investigación. Págs.1-8 Junio, 1999. http://investigacion.ilce.edu.mx/dice/articu los/articulo

${ }_{18}$ Calvo, Gloria. "El nuevo paradigma educativo: Realidad virtual vs. Aprendizaje virtual". Texto presentado en el Congreso de la Asociación Latinoamericana de Sociología -ALAS-, Chile, 1999.

${ }_{19}$ Compiladores: Jorge Orlando Castro, Myriam Henao Willes. Estado del arte de la investigación en educación y pedagogía en Colombia. Tomo II 1989-2000. Pp. 99-111. ICFES - Colciencias - SOCOLPE, 2002.
}

la ayuda de las telecomunicaciones y los satélites, el modelo educativo evolucionará a un nivel que se conoce como colaborativo: modelo en función de las ideas compartidas en grupos en discusión"20.

En relación con las prácticas pedagógicas está la concepción con que se abordan los lenguajes orales, escriturales o virtuales. En esa perspectiva Osin y Huergo (1999), cuestionan "La crisis de la lectura y la escritura, atribuida defensivamente por la escolarización a la cultura de la imagen, debería comprenderse como transformación de los modos de leer y escribir el mundo (no ya sólo el texto), como des-localización de los saberes y como desplazamiento de "lo culto" por las culturas".

Habrá que investigar si, al tener en cuenta producción, transmisión y reproducción del conocimiento, a través de los procesos de formación, enseñanza y aprendizaje, las tecnologías de la información y la comunicación, pueden ser consideradas o no como nuevas posibilidades de pensar una escuela Alternativa. Al respecto afirma Bautista: "el uso de nuevas tecnologías en las prácticas de enseñanza debe partir de temáticas que se contextualicen para los estudiantes y les involucren en discusiones que recuperen el entorno que habitan, y en esa línea les generen compromisos con su cotidianidad, les hagan responsables de las relaciones que se establecen con su entorno, con sus congéneres y consigo mismos" 21 .

La observación realizada en la Universidad permite afirmar que se percibe en la comunidad educativa la dificultad para ligar uno y otro proceso -educación y comunicación-;

\footnotetext{
${ }^{20}$ Wilches, Quintana Fabio, Profesor IPN, "El nuevo paradigma educativo: Realidad virtual vs. Aprendizaje virtual". En: Revista Colombiana de Educación.

${ }^{21}$ Bautista, Germán y otros. Tele-educación interactiva. Sistema Piloto. Convenio Colciencias - ITEC (Telecom) - UPN. 1997-2000.
} 
siguen apareciendo las incongruencias y las brechas entre una y otra categoría, a pesar de la capacidad teórica que tienen maestros y estudiantes para identificar el acto educativo como un acto que es también una acción comunicativa por excelencia.

\section{CONCLUSIONES Y PROSPECTIVA}

Como aportes de prospectiva y conclusiones, los miembros de la UPN entrevistados para la investigación de la cual, como se mencionó en la presentación, se preparó este articulo, observaron una serie de características e hicieron propuestas sobre el carácter y los propósitos que deberá tener la comunicación y uso de tecnología de la información en la Universidad, tales como:

La comunicación, como campo disciplinar, subyace en casi todos los programas y proyectos consultados, lo cual implica una validez de su necesidad e importancia pero, denota muy poca profundización temática e incipiente incorporación permanente a los procesos académicos, investigativos y de extensión. En el mejor de los casos existe la presencia de

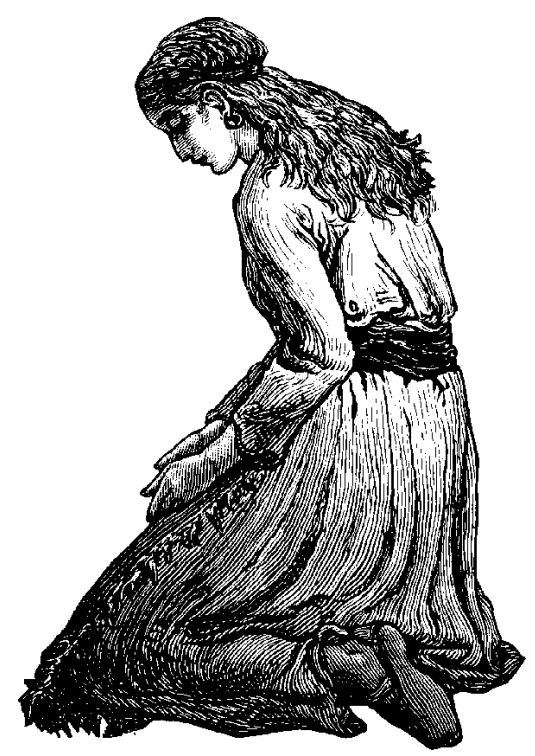

conceptos y nociones comunicativas pero se requiere incorporar la comunicación como línea transversal en las prácticas educativas.

Hay una visible preocupación por el uso de nuevas tecnologías de la información en la educación. Se observa un interesante dialogo de saberes en torno a la lectura crítico propositiva del papel de la comunicación y las tecnologías informáticas en la educación, la cultura y la sociedad, pero es necesario sistematizar el debate, sin estigmatización ni exclusión de las diferentes posiciones y tendencias que se expresan tanto al interior como al exterior de la Universidad.

La Universidad viene adelantando importantes esfuerzos por impulsar los procesos comunicativos pero se percibe dispersión y fragmentación en la construcción de líneas y políticas de investigación en torno a la relación de la comunicación y las tecnologías de la investigación en general, y en particular de la relación de esta disciplina con otros campos del conocimiento. Al respecto, se requiere adelantar consultas y explorar con distintos estamentos de la comunidad universitaria las diversas experiencias que se desarrollan para tener unas proyecciones de conjunto. En este sentido, es preciso proyectar la Universidad, a mediano plazo, en su aporte a la interpretación y transformación de las realidades educativas y socio políticas del país.

Aunque se conoce el gran impacto de la influencia que los medios de comunicación y las tecnologías de la información están ejerciendo en el ámbito educativo y en toda la sociedad, y no obstante que se hacen esfuerzos de capacitación en estas materias, es muy escasa la presencia y participación que tienen los estudiantes y profesores en la elaboración de formatos y contenidos comunicativos en las prácticas pedagógicas y en la vida universitaria. Especial énfasis necesita la formación e investigación en radio, televisión y diseño de software educativo.
En los procesos de comunicación e investigación se nota un vacío de cooperación y coordinación, una desconexión, entre los diferentes programas y proyectos, entre facultades e instancias administrativas, lo que hace que no se perciba una visión clara y especifica de los rumbos de construcción del conocimiento y su respectiva integralidad.

Se requiere fortalecer la publicación y socialización de los resultados de las investigaciones e innovaciones en revistas y eventos nacionales e internacionales con publicaciones escritas, radiales y audiovisuales que lleguen a las poblaciones de interés.

\section{Bibliografía}

Alfaro, Rosa María. "Culturas populares y comunicación participativa: en la ruta de las redefiniciones". En: Periferia. Revista de comunicación y estudios culturales. No. 2 Neiva, 1999.

Ávila Penagos, Rafael. "La construcción social de los proyectos educativos institucionales": En: La investigación en Educación y Pedagogía. Bogotá, 1999.

BRUNNER, José Joaquín. Educación superior en América Latina: una agenda para el año 2000. Bogotá, 1995.

Castro, Jorge Orlando; Henao, Willes Myriam, (compiladores) Estado del arte de la investigación en educación y pedagogía en Colombia. 1989-2001. Tomo II. ICFES-COLCIENCIAS-SOCOLPE. 2003.

CENECA - UNICEF - UNESCO Educación para la comunicación. Manual latinoamericano de educación para los medios de comunicación. Quito, 1992.

De OLIVEIRA, Soares Ismar. "Comunicación / educación: itinerarios transversales". En: Seminario Internacional de Comunicación / educación en América Latina, Bogotá, 1999.

Delors, Jacques. La educación encierra un tesoro. Madrid, UNESCO, 1996.

Fals Borda, Orlando y Rahman, Anizur (Compiladotes). Acción y conocimiento. 
Cómo romper el monopolio con la investigación acción participación, CINEP, Bogotá, $232 \mathrm{p}$.

Galtung, Johan. Después del proyecto. Camelot, 1998.

Gómez, Víctor Manuel. Educadores e informática: Promesas, dilemas y realidades. Bogotá, 1997.

GómEz, Hernando. "Lineamientos pedagógicos para una educación por competencias". En: Bustamante, Guillermo. El concepto de competencia II. Una Mirada in terdisciplinaria. Sociedad Colombiana de Pedagogía, Alejandría Libros: 2002. Págs. 145-153-154-15.

GómEz, Pablo Gilberto. En: Seminario internacional de comunicación /educación en América Latina 1ํ: 1999, Bogotá, 1999.

GonzÁlez, Cristóbal. Comunicación para un nuevo país en paz. Bogotá, Universidad Incca, 2000.

Henao, Myriam, Tamayo Valencia, Alfonso. Políticas públicas y universidad. Bogotá, 1999.

Hernández, Carlos Augusto. Universidad Nacional de Colombia. "Aproximación a un estado del arte de la enseñanza de las ciencias en Colombia". COLCIENCIAS e ICFES, 2001, págs. 14-15-16.

Herrán, María Teresa. La escuela y los medios masivos de comunicación, Bogotá, 1997.
Huergo, Jorge Alberto; Osin, Luis. Comunicación, humanismo y nuevas tecnologías en el espacio escolar. CACE-UPN, pág. 155.

Huergo, Jorge Alberto. "Comunicación/educación: itinerarios transversales". En: Seminario internacional de comunicación/ educación en América Latina 1ํ. Bogotá, 1999.

KAPLUN, Mario. El comunicador popular. Quito, 1985.

LEWIS, Peter. "Informe sobre la comunicación en el mundo. Medios de Comunicación alternativos: la conexión mundial con lo local". En: Estudios y documentos de comunicación de masas. París, 1995.

Martín-Barbero, Jesús. "Heredando el futuro. Pensar la educación desde la comunicación". En: Revista Nómadas No. 5, Bogotá, Universidad Central, 1996, pp. 23-32.

MatTelard, Armand. Tecnología, cultura y comunicación. Barcelona, 1984.

Pascali, Antonio. Comunicación, culturay masa. Bogotá, 1990.

Parra, Rodrigo; Cajiao, Francisco. Génesis: investigación sobre el papel de las innovaciones en la modernización.

REY, Paola Inés. "Nuevas tecnologías aplicadas a la educación. Estado del arte de la investigación 1990-1999". En: Estado del Arte de la Investigación en Educación y
Pedagogía en Colombia II. COLCIENCIAS, ICFES, SOCOLPE. 2001, pág. 181.

SÁncHeZ, Jesús Alberto. Apropiación pedagógica del campo intelectual de la educación para la construcción de un modelo comprensivo de la formación de docentes. ACIFORMA, Medellín, 1996.

SANDOVAL, Sandra. La función de educadores en Colombia, tomos I y II. Compilación, Bogotá, 2001.

Stolowicz, Beatriz. Universidad Autónoma Metropolitana. Unidad, México, D.F. V Encuentro Latinoamericano de Sociología. Bogotá, abril 2001: 3.

Tedesco, Juan Carlos. "Algunas hipótesis para una política de innovaciones educativas". En: Proyecto Génesis. Innovación escolar y cambio social. Tomo I. Bogotá, 1997.

TOURAINE, Alain ¿Podremos vivir juntos? La discusión pendiente: el destino del hombre en la aldea global. Buenos Aires, F.C.E., 1997.

TrianA Nova, Betty María. Interés y comunicación en el proceso de enseñanza y aprendizaje. Hemeroteca UPN., 1999.

VAlderRama, Carlos. "Introducción a comunicación/educación: Coordenadas, abordajes y travesías. Bogotá, Universidad Central, 2000.

VÁsQUEZ, Tomás. Educación y medios masivos de comunicación. Bogotá, UPN, 1990.

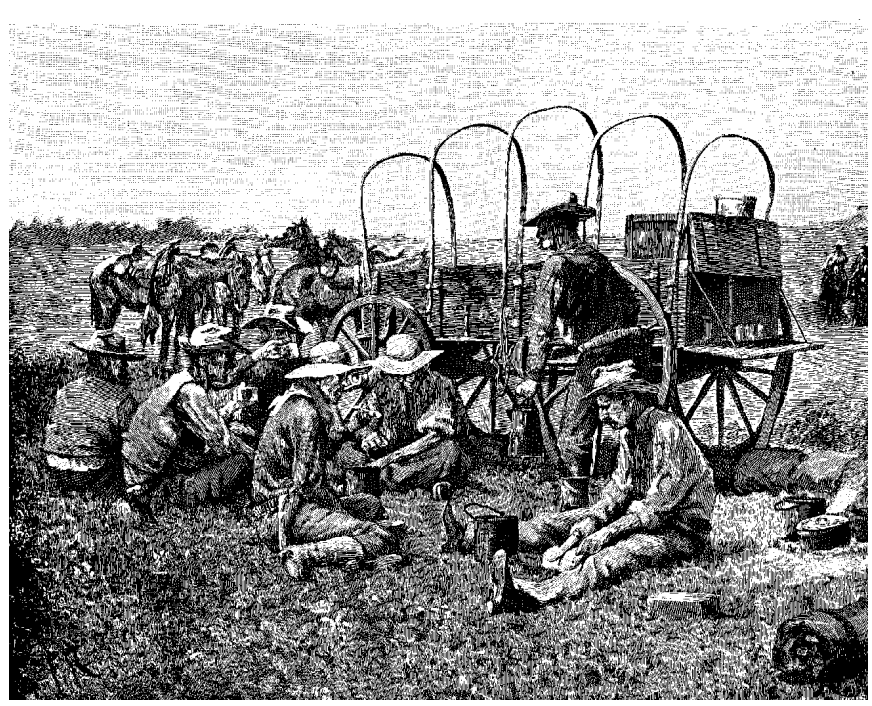

